\title{
Long-Term Effect of Manures and Fertilizers on Nutrient Status under Cotton Mono-Cropping in Vertisol
}

\author{
P. Joga Rao ${ }^{1 *}$, P.R.K. Prasad ${ }^{1}$, A. Lalitha Kumari ${ }^{2}$, P. Prasuna Rani ${ }^{3}$ and C.H. Pulla Rao ${ }^{4}$ \\ ${ }^{1}$ Agricultural College, Bapatla, ANGRAU, AP, India \\ ${ }^{2}$ Regional Research Unit, RARS, Lam, ANGRAU, Guntur, AP, India \\ ${ }^{3}$ Saline Water Scheme, Bapatla, ANGRAU, Guntur, AP, India \\ ${ }^{4}$ Department of Agronomy, Agricultural College, Bapatla, ANGRAU, AP, India \\ *Corresponding author
}

\begin{abstract}
A B S T R A C T
Keywords

Cotton, Vertisol, Long-term effect, Soil properties, Seed cotton yield.

Article Info

Accepted:

21 June 2017

Available Online:

10 July 2017

The status of nutrients-their depletion and build up in soil and crop productivity after twenty two years (1991-2012) of cotton mono-cropping were studied under continuous use of various inorganic fertilizers and organic manure in a Vertisol. Results showed that application of $100 \%$ RD of NPK (90:45:45 kg ha $\left.{ }^{-1}\right)+F Y M @ 10 \mathrm{t} \mathrm{ha}^{-1}$ recorded highest seed cotton yield of $2181 \mathrm{~kg} \mathrm{ha}^{-1}$ which was on par with $150 \%$ RD of NPK treated plot. The soil $\mathrm{pH}$ and EC did not change significantly but markedly changed the organic carbon and available nutrient contents of the soil. Thus, the balanced use of fertilizers continuously either alone or in combination with organic manure is necessary for sustaining soil fertility and productivity of cotton under rainfed conditions.
\end{abstract}

\section{Introduction}

Cotton is the most important fiber crop of the world. It is the most beneficial fiber and cash crop of India and earns a good fortune for the country in the form of foreign exchange (Ahmed et al., 2009). Increased nitrogen rate resulting in increased biological yield may be due to increase in $\mathrm{N}$ rate and increases mineral uptake, photosynthetic assimilation and accumulation in sinks Sawan et al., (2006). The decline in soil fertility due to imbalanced fertilizer use has been recognized as one of the most important factor limiting crop yields (Nambiar et al., 1989). Decline in yield has been observed in many cropping systems in many parts of the country due to nutrient depletion, soil structure deterioration and imbalanced use of plant nutrients, acidification, and sub-optimal addition of organic and inorganic fertilizers to soil. The capacity of soil for sustaining production depends on its fertility status. Organic matter helps in increasing adsorptive power of soil for nutrient ions. These adsorbed nutrient ions are released slowly for the benefit of crop during entire growth period. It helps to improve the soil physical (Biswas and Khosla, 1971) and chemical properties. Organic manures can play an important role in 
sustaining the productivity by not only acting as a source of nutrients but also through modifying soil physical behavior as well as increasing the efficiency of applied nutrients (Sahadevareddy and Aruna, 2008).

\section{Materials and Methods}

The long-term fertilizer experiment was initiated during the season kharif 1991 at Regional Agricultural Research Station, Lam, Guntur, Andhra Pradesh. The present investigation was carried out during the season kharif 2013-14 on Vertisol soil after 22 years of this experimentation in the same field. The experiment involves 11 treatments each replicated three times in a randomized block design. The test crop was cotton, variety L-799. The crop was sown on 13-082013 and harvested on 09-03-2014 (Final picking). The initial (1991-92) soil characters were as following. The soil $\mathrm{pH} 8.4$, E.C. 0.60 $\mathrm{dSm}^{-1}$, organic carbon $0.37 \%$ and $\mathrm{N}, \mathrm{P}_{2} \mathrm{O}_{5}$, $\mathrm{K}_{2} \mathrm{O} 196,23$ and $392 \mathrm{~kg} \mathrm{ha}^{-1}$, respectively (Table 10). Cotton was raised during kharif 2013-14 adopting recommended package of practices. The recommended fertilizers (90:45:45 kg N, $\mathrm{P}_{2} \mathrm{O}_{5}$ and $\mathrm{K}_{2} \mathrm{O}$ ha $^{-1}$ ) were applied through urea $(46 \% \mathrm{~N})$, single superphosphate $\left(16 \% \mathrm{P}_{2} \mathrm{O}_{5}\right)$ and muriate of potash $\left(60 \% \mathrm{~K}_{2} \mathrm{O}\right)$, as per the treatments. FYM was applied 10 days before sowing in the respective treatments. Phosphorus, $\mathrm{ZnSO}_{4}$, $\mathrm{MgSO}_{4}$ and gypsum application was done before sowing. Nitrogen and potassium fertilizers were applied in three splits. Soil samples were collected before sowing of the crop at two depths i.e., 0-15 and 15-30 cm.

Soil reaction was determined in 1: 2.5 soil water suspension using combined glass electrode (Jackson, 1973). The E.C of soil samples was determined in 1: 2.5 soil water extract using electrical conductivity bridge (Jackson, 1973). Walkley and Black's "wet digestion method" as outlined by Jackson
(1973) was followed to determine the organic carbon content of the soils. Available nitrogen content in the soils was determined by alkaline permanganate method (Subbiah and Asija, 1956). Available phosphorus in the soil samples was extracted with $0.5 \mathrm{M} \mathrm{NaHCO}_{3}$ of pH 8.5 (Olsen et al., 1954). Available potassium in soil was extracted using neutral normal ammonium acetate and potassium in the extract was determined flame photometrically gien by Muhr et al., 1965 .

The content of nitrogen in cotton plants was estimated by micro Kjeldahl method (Piper, 1966). Phosphorus in the di-acid extract of plant samples was estimated by vanado molybdo phosphoric yellow colour method using spectrophotometer at $420 \mathrm{~nm}$ as described by Jackson (1973). Potassium in the di-acid extract of plant sample was determined using flame photometer as per the method described by Jackson (1973).

\section{Results and Discussion}

\section{Soil pH}

The $\mathrm{pH}$ of the soil was not influenced at different stages (initial, flowering and harvest) of crop growth by different treatments in both the surface and subsurface soil. A glance of the data in table 1 revealed that soil $\mathrm{pH}$ was slightly increased from initial to harvest stage. There was a reduction in soil $\mathrm{pH}$ when compared to the initial (1991-92) value of 8.4. However, the treatment differences were non-significant.

Irrespective of nutrient management provided to cotton crop, the soil $\mathrm{pH}$ at different stages of crop growth decreased with increasing level of NPK (Table 1) from 50 to $200 \%$ but not at significant level. Those findings were in consonance with the work of Sinha et al., (1997) and Prasad et al., (2010a) who reported that the continuous use of urea 
fertilizer to both crops could able to decrease the $\mathrm{pH}$ after harvest of maize crop.

\section{Electrical conductivity $\left(\mathrm{dS} \mathrm{m} \mathbf{m}^{-1}\right)$}

The EC of the soil was not significantly influenced by different treatments in the surface and subsurface soil. The EC of surface and subsurface soils ranged from 0.18 to $0.30 \mathrm{dS} \mathrm{m}^{-1}$ at various stages. The highest $0.30 \mathrm{dS} \mathrm{m}^{-1}$ was recorded in $200 \% \mathrm{RD}$ of NPK $\left(\mathrm{T}_{10}\right)$ initial at surface soils and the lowest value $0.18 \mathrm{dS} \mathrm{m}^{-1}$ was recorded in the treatment control $\left(\mathrm{T}_{1}\right)$ harvest at subsurface soils. The trend of variation in EC of the soil between the treatments in both the soil layers was almost negligible and statistically nonsignificant (Table 2). There was considerable decrease in the soluble salt content of soil which was relatively less compared to the initial (1991-92) value of $0.60 \mathrm{dS} \mathrm{m}^{-1}$.

The electrical conductivity decreased from initial to harvest stage in all the experimental treatments in surface and sub-surface soils. The heavy root system of the cotton makes the soil loose and porous, so the salts added on addition of chemical fertilizers might have leached away readily resulting in low EC in cotton growing soils. Low electrical conductivity in cotton growing soils, attesting to intense ion leaching was reported by Giora et al., (2010).

\section{Soil organic carbon}

The glance of the data (Table 3, Fig. 1) revealed that organic carbon content increased from initial to flowering stage and it was decreased at harvest in all the experimental treatments in surface as well as in sub-surface soils. An overall increase in organic carbon content was observed in the present study under all the treatments as compared to their initial status of organic carbon. Increase in recommended level of NPK from 50 to 150 per cent $\left(T_{2}, T_{3}\right.$ and $\left.T_{4}\right)$, organic carbon content was gradually increased up to 150 per cent and decreased at $200 \%$ RD of NPK $\left(T_{10}\right)$.

The highest value $0.61 \%$ (flowering stage, surface soils) was observed in FYM @ $10 \mathrm{t}$ ha $^{-1}$ along with $100 \%$ RD of NPK treated plot $\left(\mathrm{T}_{7}\right)$ followed by $150 \%$ of RD of NPK treated plot $\left(\mathrm{T}_{4}\right)$ and $200 \%$ RD of NPK treated plot $\left(\mathrm{T}_{11}\right)$ whereas the lowest value $0.33 \%$ (initial, subsurface soil) was observed in control $\left(\mathrm{T}_{1}\right)$ (Table 3). The increase in organic carbon content in the surface soil as compared to the subsurface soil was mainly due to the accumulation of organic residues over a period of time.

\section{Available nitrogen}

The highest value $\left(202 \mathrm{~kg} \mathrm{ha}^{-1}\right)$ of available nitrogen was recorded with $100 \%$ RD of NPK + FYM @ $10 \mathrm{t} \mathrm{ha}^{-1}\left(\mathrm{~T}_{7}\right)$ treated plot (flowering stage, surface soil) followed by $150 \% \mathrm{RD}$ of NPK $\left(\mathrm{T}_{4}\right)$ treated plot $(198 \mathrm{~kg}$ $\mathrm{ha}^{-1}$ ) (flowering stage, surface soil) followed by chemical fertilizers (100\% RD of NPK) with the combination of gypsum $\left(\mathrm{T}_{11}\right)$, Zinc $\left(\mathrm{T}_{8}\right)$ and $\mathrm{MgSO}_{4}\left(\mathrm{~T}_{9}\right)$ (Table 4$)$. The highest value of available $\mathrm{N}$ due to incorporation of FYM $10 \mathrm{t} \mathrm{ha}^{-1}$ along with balanced fertilizer application once in year over a period of time might be attributed to enhanced mineralization and accumulation of $\mathrm{N}$ in surface soil layer. The surface soil samples had relatively high nitrogen content compared to sub-surface soils (Table 4, Fig. 2). The decrease of nitrogen availability with depth might be due to the low organic matter in the sub-surface (Bandyopadhyay et al., 2004).

\section{Available phosphorus}

The available phosphorus content of soil also increased with maturity of the crop and similar to available nitrogen. Table 5 and figure 3 showed that there was a build-up of available $\mathrm{P}_{2} \mathrm{O}_{5}$ (except $\mathrm{T}_{1}$ and $\mathrm{T}_{6}$ ) over the initial value of $23 \mathrm{~kg} \mathrm{P}_{2} \mathrm{O}_{5} \mathrm{ha}^{-1}$. Application of 
FYM @ 10 t ha ${ }^{-1}$ along with $\operatorname{RDF}\left(\mathrm{T}_{7}\right)$ recorded the highest amount of available phosphorus at initial, flowering and harvest as compared to rest of the treatments while, the lowest was recorded with $\mathrm{T}_{1}$ (control). The available $\mathrm{P}$ was higher in the surface soils compared to sub-surface soils.

The highest available $\mathrm{P}_{2} \mathrm{O}_{5}$ was observed in $\mathrm{T}_{7}$ treatment. Organic matter on the surface favoured the solubilisation of insoluble phosphorus releasing more quantity to the surface (Chaudhary et al., 2006) and also due to the confinement of crop cultivation to the rhizosphere and supplementing of the depleted phosphorus through external sources i.e., fertilizers.

\section{Available potassium}

Available K content of the soil increased (Table 6, Fig. 4) in all the treatments studied during kharif 2013-14 compared to the initial status (392 kg K ha ${ }^{-1}$ ) of the year 1991. Surface soil samples had higher available potassium content when compared to subsurface soil. This could be due to more intense weathering, release of $\mathrm{K}$ from organic residues, application of $\mathrm{K}$ fertilizers and upward translocation of potassium from lower depth along with capillary raise of ground water (Hirekurabar et al., 2000).

\section{Seed cotton yield}

The data represented in table 7 and figure 5 indicated that among the treatments, FYM treated plot $\left(\mathrm{T}_{7}, \mathrm{RDF}+\mathrm{FYM} @ 10 \mathrm{t} \mathrm{ha}^{-1}\right)$ recorded significant increase in seed cotton yield along with $\mathrm{T}_{4}(150 \%$ of $\mathrm{RDF})$ over $\mathrm{T}_{3}$ (100\% RDF). The comparison of treatment $\mathrm{T}_{3}$ (100\% RDF) with $\mathrm{T}_{8}$ (100 per cent RD of $\left.\mathrm{NPK}+\mathrm{ZnSO}_{4} @ 50 \mathrm{~kg} \mathrm{ha}^{-1}\right), \mathrm{T}_{9}(100 \% \mathrm{RD}$ of $\left.\mathrm{NPK}+\mathrm{MgSO}_{4} @ 50 \mathrm{~kg} \mathrm{ha}^{-1}\right)$ and $\mathrm{T}_{11}(100 \%$ $\mathrm{RD}$ of NPK + gypsum @ $5 \mathrm{q} \mathrm{ha}^{-1}$ ), did not show any marked effect on seed cotton yield. The yield reduction was 11.9 and 19.4 per cent in $\mathrm{T}_{5}$ and $\mathrm{T}_{6}$, respectively over $\mathrm{T}_{3}$.

The highest seed cotton yield was observed in $\mathrm{T}_{7}$ treatment, due to the use of organic manures like FYM. It attributed to increased microbial activity which in turn helped in transformation of nutrients making them more available to plants. Similar observations were reported by Lalithakumari et al., (2010).

Table.1 Effect of long-term use of manures and fertilizers on soil $\mathrm{pH}$

\begin{tabular}{|c|c|c|c|c|c|c|}
\hline \multirow{2}{*}{ Treatments } & \multicolumn{3}{|c|}{ Surface } & \multicolumn{3}{|c|}{ Sub-surface } \\
\hline & Initial & Flowering & Harvest & Initial & Flowering & Harvest \\
\hline $\mathrm{T}_{1}$ : Control & 8.3 & 8.4 & 8.4 & 8.3 & 8.3 & 8.3 \\
\hline $\mathrm{T}_{2}: 50 \% \mathrm{RD}$ of NPK & 8.3 & 8.3 & 8.3 & 8.3 & 8.3 & 8.3 \\
\hline $\mathrm{T}_{3}: 100 \% \mathrm{RD}$ of NPK & 8.3 & 8.3 & 8.3 & 8.2 & 8.3 & 8.3 \\
\hline $\mathrm{T}_{4}: 150 \% \mathrm{RD}$ of NPK & 8.3 & 8.3 & 8.3 & 8.2 & 8.3 & 8.3 \\
\hline $\mathrm{T}_{5}: 100 \% \mathrm{RD}$ of NP & 8.2 & 8.3 & 8.3 & 8.2 & 8.2 & 8.2 \\
\hline $\mathrm{T}_{6}: 100 \% \mathrm{RD}$ of $\mathrm{N}$ & 8.2 & 8.2 & 8.2 & 8.1 & 8.2 & 8.1 \\
\hline $\mathrm{T}_{7}: \mathrm{T}_{3}+\mathrm{FYM} @ 10 \mathrm{tha}^{-1}$ & 8.1 & 8.1 & 8.2 & 8.0 & 8.1 & 8.0 \\
\hline $\mathrm{T}_{8}: \mathrm{T}_{3}+\mathrm{ZnSO}_{4} @ 50 \mathrm{~kg} \mathrm{ha}^{-1}$ & 8.2 & 8.3 & 8.3 & 8.2 & 8.3 & 8.2 \\
\hline $\mathrm{T}_{9}: \mathrm{T}_{3}+\mathrm{MgSO}_{4} @ 50 \mathrm{~kg} \mathrm{ha}^{-1}$ & 8.2 & 8.3 & 8.3 & 8.2 & 8.2 & 8.3 \\
\hline $\mathrm{T}_{10}: 200 \% \mathrm{RD}$ of NPK & 8.2 & 8.3 & 8.3 & 8.2 & 8.2 & 8.3 \\
\hline $\mathrm{T}_{11}: \mathrm{T}_{3}+$ gypsum @ $5 \mathrm{q} \mathrm{ha}^{-1}$ & 8.2 & 8.2 & 8.2 & 8.1 & 8.1 & 8.2 \\
\hline SEm \pm & 0.24 & 0.25 & 0.27 & 0.24 & 0.24 & 0.24 \\
\hline $\mathrm{CD}(0.05)$ & $\mathrm{NS}$ & $\mathrm{NS}$ & $\mathrm{NS}$ & $\mathrm{NS}$ & $\mathrm{NS}$ & NS \\
\hline $\mathrm{CV}(\%)$ & 5.1 & 5.2 & 5.0 & 5.1 & 5.1 & 5.1 \\
\hline
\end{tabular}


Table.2 Effect of long-term use of manures and fertilizers on soil EC ( $\left.\mathrm{dS} \mathrm{m}^{-1}\right)$

\begin{tabular}{|c|c|c|c|c|c|c|}
\hline \multirow[b]{2}{*}{ Treatments } & \multicolumn{3}{|c|}{ Surface } & \multicolumn{3}{|c|}{ Sub-surface } \\
\hline & Initial & $\begin{array}{l}\text { Flowerin } \\
\text { g }\end{array}$ & Harvest & $\begin{array}{l}\text { Initia } \\
\text { l }\end{array}$ & $\begin{array}{l}\text { Flowerin } \\
\text { g }\end{array}$ & Harvest \\
\hline $\mathrm{T}_{1}:$ Control & 0.21 & 0.20 & 0.19 & 0.20 & 0.19 & 0.18 \\
\hline $\mathrm{T}_{2}: 50 \% \mathrm{RD}$ of $\mathrm{NPK}$ & 0.26 & 0.25 & 0.24 & 0.24 & 0.23 & 0.23 \\
\hline $\mathrm{T}_{3}: 100 \% \mathrm{RD}$ of NPK & 0.28 & 0.26 & 0.25 & 0.26 & 0.25 & 0.24 \\
\hline $\mathrm{T}_{4}: 150 \% \mathrm{RD}$ of $\mathrm{NPK}$ & 0.29 & 0.27 & 0.26 & 0.27 & 0.26 & 0.25 \\
\hline $\mathrm{T}_{5}: 100 \% \mathrm{RD}$ of NP & 0.27 & 0.26 & 0.25 & 0.25 & 0.25 & 0.24 \\
\hline $\mathrm{T}_{6}: 100 \% \mathrm{RD}$ of $\mathrm{N}$ & 0.26 & 0.24 & 0.23 & 0.25 & 0.23 & 0.23 \\
\hline $\mathrm{T}_{7}: \mathrm{T}_{3}+\mathrm{FYM} @ 10 \mathrm{tha}^{-1}$ & 0.23 & 0.22 & 0.21 & 0.22 & 0.21 & 0.20 \\
\hline $\mathrm{T}_{8}: \mathrm{T}_{3}+\mathrm{ZnSO}_{4} @ 50 \mathrm{~kg} \mathrm{ha}^{-1}$ & 0.29 & 0.26 & 0.25 & 0.27 & 0.25 & 0.25 \\
\hline $\mathrm{T}_{9}: \mathrm{T}_{3}+\mathrm{MgSO}_{4} @ 50 \mathrm{~kg} \mathrm{ha}^{-1}$ & 0.27 & 0.26 & 0.25 & 0.26 & 0.25 & 0.24 \\
\hline $\mathrm{T}_{10}: 200 \% \mathrm{RD}$ of NPK & 0.30 & 0.29 & 0.28 & 0.29 & 0.27 & 0.27 \\
\hline $\mathrm{T}_{11}: \mathrm{T}_{3}+$ gypsum @ $5 \mathrm{q} \mathrm{ha}^{-1}$ & 0.24 & 0.23 & 0.22 & 0.22 & 0.22 & 0.21 \\
\hline SEm \pm & 0.01 & 0.01 & 0.02 & 0.01 & 0.01 & 0.01 \\
\hline $\mathrm{CD}(0.05)$ & NS & NS & $\mathrm{NS}$ & $\mathrm{NS}$ & $\mathrm{NS}$ & $\mathrm{NS}$ \\
\hline $\mathrm{CV}(\%)$ & 8.1 & 7.2 & 12.7 & 7.5 & 10.8 & 10.4 \\
\hline
\end{tabular}

Table.3 Effect of long-term use of manures and fertilizers on soil organic carbon content (\%)

\begin{tabular}{|c|c|c|c|c|c|c|}
\hline \multirow[b]{2}{*}{ Treatments } & \multicolumn{3}{|c|}{ Surface } & \multicolumn{3}{|c|}{ Sub-surface } \\
\hline & Initial & Flowering & Harvest & Initial & Flowering & Harvest \\
\hline $\mathrm{T}_{1}:$ Control & 0.34 & 0.37 & 0.36 & 0.33 & 0.36 & 0.35 \\
\hline $\mathrm{T}_{2}: 50 \% \mathrm{RD}$ of $\mathrm{NPK}$ & 0.36 & 0.40 & 0.38 & 0.35 & 0.39 & 0.37 \\
\hline $\mathrm{T}_{3}: 100 \% \mathrm{RD}$ of NPK & 0.45 & 0.48 & 0.48 & 0.44 & 0.47 & 0.47 \\
\hline $\mathrm{T}_{4}: 150 \% \mathrm{RD}$ of NPK & 0.56 & 0.60 & 0.56 & 0.53 & 0.56 & 0.54 \\
\hline $\mathrm{T}_{5}: 100 \% \mathrm{RD}$ of NP & 0.44 & 0.48 & 0.47 & 0.43 & 0.47 & 0.45 \\
\hline $\mathrm{T}_{6}: 100 \% \mathrm{RD}$ of $\mathrm{N}$ & 0.43 & 0.48 & 0.46 & 0.42 & 0.46 & 0.45 \\
\hline $\mathrm{T}_{7}: \mathrm{T}_{3}+\mathrm{FYM} @ 10 \mathrm{tha}^{-1}$ & 0.58 & 0.61 & 0.59 & 0.56 & 0.58 & 0.57 \\
\hline $\mathrm{T}_{8}: \mathrm{T}_{3}+\mathrm{ZnSO}_{4} @ 50 \mathrm{~kg} \mathrm{ha}^{-1}$ & 0.47 & 0.50 & 0.49 & 0.45 & 0.48 & 0.48 \\
\hline $\mathrm{T}_{9}: \mathrm{T}_{3}+\mathrm{MgSO}_{4} @ 50 \mathrm{~kg} \mathrm{ha}^{-1}$ & 0.46 & 0.49 & 0.48 & 0.45 & 0.47 & 0.47 \\
\hline $\mathrm{T}_{10}: 200 \% \mathrm{RD}$ of NPK & 0.52 & 0.55 & 0.53 & 0.51 & 0.53 & 0.51 \\
\hline $\mathrm{T}_{11}: \mathrm{T}_{3}+$ gypsum @ $\mathrm{q} \mathrm{ha}^{-1}$ & 0.48 & 0.52 & 0.49 & 0.46 & 0.49 & 0.49 \\
\hline SEm \pm & 0.02 & 0.02 & 0.02 & 0.02 & 0.02 & 0.03 \\
\hline $\mathrm{CD}(0.05)$ & 0.07 & NS & 0.05 & 0.06 & 0.07 & 0.08 \\
\hline $\mathrm{CV}(\%)$ & 8.4 & 5.5 & 6.6 & 7.3 & 8.4 & 9.6 \\
\hline
\end{tabular}


Table.4 Effect of long-term use of manures and fertilizers on soil Available nitrogen content $\left(\mathrm{kg} \mathrm{ha}^{-1}\right)$

\begin{tabular}{|c|c|c|c|c|c|c|}
\hline \multirow{2}{*}{ Treatments } & \multicolumn{3}{|c|}{ Surface } & \multicolumn{3}{|c|}{ Sub-surface } \\
\hline & Initial & Flowering & Harvest & Initial & Flowering & Harvest \\
\hline $\mathrm{T}_{1}:$ Control & 154 & 165 & 159 & 151 & 163 & 154 \\
\hline $\mathrm{T}_{2}: 50 \% \mathrm{RD}$ of $\mathrm{NPK}$ & 159 & 176 & 162 & 157 & 173 & 157 \\
\hline $\mathrm{T}_{3}: 100 \% \mathrm{RD}$ of $\mathrm{NPK}$ & 163 & 180 & 167 & 161 & 176 & 164 \\
\hline $\mathrm{T}_{4}: 150 \% \mathrm{RD}$ of NPK & 172 & 198 & 181 & 171 & 192 & 178 \\
\hline $\mathrm{T}_{5}: 100 \% \mathrm{RD}$ of $\mathrm{NP}$ & 161 & 179 & 166 & 160 & 176 & 162 \\
\hline $\mathrm{T}_{6}: 100 \% \mathrm{RD}$ of $\mathrm{N}$ & 161 & 178 & 165 & 158 & 175 & 159 \\
\hline $\mathrm{T}_{7}: \mathrm{T}_{3}+\mathrm{FYM} @ 10 \mathrm{tha}^{-1}$ & 182 & 202 & 189 & 181 & 200 & 184 \\
\hline $\mathrm{T}_{8}: \mathrm{T}_{3}+\mathrm{ZnSO}_{4} @ 50 \mathrm{~kg} \mathrm{ha}^{-1}$ & 163 & 182 & 169 & 162 & 180 & 164 \\
\hline $\mathrm{T}_{9}: \mathrm{T}_{3}+\mathrm{MgSO}_{4} @ 50 \mathrm{~kg} \mathrm{ha}^{-1}$ & 163 & 182 & 169 & 162 & 178 & 164 \\
\hline $\mathrm{T}_{10}: 200 \% \mathrm{RD}$ of NPK & 168 & 186 & 175 & 164 & 184 & 173 \\
\hline $\mathrm{T}_{11}: \mathrm{T}_{3}+$ gypsum @ $9 \mathrm{q} \mathrm{ha}^{-1}$ & 167 & 185 & 173 & 164 & 182 & 172 \\
\hline SEm \pm & 5.11 & 5.42 & 4.24 & 5.22 & 5.54 & 5.20 \\
\hline $\mathrm{CD}(0.05)$ & 15.1 & 15.9 & 12.5 & $\mathrm{NS}$ & 16.3 & 15.3 \\
\hline $\mathrm{CV}(\%)$ & 5.9 & 5.1 & 5.2 & 5.5 & 5.3 & 5.4 \\
\hline
\end{tabular}

Table.5 Effect of long-term use of manures and fertilizers on soil Available phosphorus $\left(\mathrm{kg} \mathrm{P}_{2} \mathrm{O}_{5} \mathrm{ha}^{-1}\right)$

\begin{tabular}{|c|c|c|c|c|c|c|}
\hline \multirow{2}{*}{ Treatments } & \multicolumn{3}{|c|}{ Surface } & \multicolumn{3}{|c|}{ Sub-surface } \\
\hline & Initial & Flowering & Harvest & Initial & Flowering & Harvest \\
\hline $\mathrm{T}_{1}$ : Control & 20.4 & 21.2 & 20.6 & 17.5 & 20.0 & 19.7 \\
\hline $\mathrm{T}_{2}: 50 \%$ RD of NPK & 33.8 & 41.2 & 38.2 & 31.1 & 38.0 & 36.7 \\
\hline $\mathrm{T}_{3}: 100 \% \mathrm{RD}$ of NPK & 36.3 & 47.7 & 43.3 & 33.8 & 44.6 & 41.1 \\
\hline $\mathrm{T}_{4}: 150 \% \mathrm{RD}$ of NPK & 45.4 & 52.8 & 48.6 & 42.9 & 49.5 & 46.0 \\
\hline $\mathrm{T}_{5}: 100 \% \mathrm{RD}$ of NP & 36.0 & 46.5 & 43.2 & 34.6 & 44.7 & 40.6 \\
\hline $\mathrm{T}_{6}: 100 \% \mathrm{RD}$ of $\mathrm{N}$ & 21.9 & 23.5 & 22.2 & 19.0 & 22.1 & 20.4 \\
\hline $\mathrm{T}_{7}: \mathrm{T}_{3}+\mathrm{FYM} @ 10 \mathrm{t} \mathrm{ha}^{-1}$ & 51.8 & 62.8 & 56.4 & 48.2 & 60.8 & 52.9 \\
\hline $\mathrm{T}_{8}: \mathrm{T}_{3}+\mathrm{ZnSO}_{4} @ 50 \mathrm{~kg} \mathrm{ha}^{-1}$ & 37.3 & 49.3 & 43.6 & 35.8 & 44.9 & 42.2 \\
\hline $\mathrm{T}_{9}: \mathrm{T}_{3}+\mathrm{MgSO}_{4} @ 50 \mathrm{~kg} \mathrm{ha}^{-1}$ & 37.4 & 49.1 & 43.8 & 36.4 & 45.0 & 41.5 \\
\hline $\mathrm{T}_{10}: 200 \% \mathrm{RD}$ of NPK & 48.4 & 56.5 & 53.0 & 43.4 & 52.6 & 50.6 \\
\hline $\mathrm{T}_{11}: \mathrm{T}_{3}+$ gypsum @ $5 \mathrm{q} \mathrm{ha}^{-1}$ & 40.9 & 51.5 & 48.2 & 39.5 & 49.3 & 45.5 \\
\hline SEm \pm & 2.3 & 2.1 & 2.3 & 1.7 & 2.2 & 2.5 \\
\hline $\mathrm{CD}(0.05)$ & 6.7 & 6.3 & 6.8 & 4.9 & 6.6 & 7.4 \\
\hline $\mathrm{CV}(\%)$ & 10.6 & 8.1 & 9.5 & 8.3 & 9.0 & 10.9 \\
\hline
\end{tabular}


Table.6 Effect of long-term use of manures and fertilizers on soil Available potassium $\left(\mathrm{kg} \mathrm{K}_{2} \mathrm{O} \mathrm{ha}^{-1}\right)$

\begin{tabular}{|c|c|c|c|c|c|c|}
\hline \multirow{2}{*}{ Treatments } & \multicolumn{3}{|c|}{ Surface } & \multicolumn{3}{|c|}{ Sub-surface } \\
\hline & Initial & Flowering & Harvest & Initial & Flowering & Harvest \\
\hline $\mathrm{T}_{1}:$ Control & 461 & 480 & 470 & 448 & 465 & 459 \\
\hline $\mathrm{T}_{2}: 50 \% \mathrm{RD}$ of $\mathrm{NPK}$ & 512 & 522 & 515 & 496 & 492 & 503 \\
\hline $\mathrm{T}_{3}: 100 \% \mathrm{RD}$ of $\mathrm{NPK}$ & 549 & 559 & 530 & 533 & 526 & 517 \\
\hline $\mathrm{T}_{4}: 150 \% \mathrm{RD}$ of NPK & 628 & 663 & 644 & 621 & 633 & 633 \\
\hline $\mathrm{T}_{5}: 100 \% \mathrm{RD}$ of $\mathrm{NP}$ & 535 & 537 & 528 & 506 & 521 & 512 \\
\hline $\mathrm{T}_{6}: 100 \% \mathrm{RD}$ of $\mathrm{N}$ & 479 & 515 & 510 & 456 & 507 & 482 \\
\hline $\mathrm{T}_{7}: \mathrm{T}_{3}+\mathrm{FYM} @ 10 \mathrm{tha}^{-1}$ & 695 & 712 & 664 & 679 & 649 & 648 \\
\hline $\mathrm{T}_{8}: \mathrm{T}_{3}+\mathrm{ZnSO}_{4} @ 50 \mathrm{~kg} \mathrm{ha}^{-1}$ & 558 & 570 & 532 & 542 & 535 & 520 \\
\hline $\mathrm{T}_{9}: \mathrm{T}_{3}+\mathrm{MgSO}_{4} @ 50 \mathrm{~kg} \mathrm{ha}^{-1}$ & 563 & 580 & 555 & 549 & 524 & 537 \\
\hline $\mathrm{T}_{10}: 200 \% \mathrm{RD}$ of NPK & 618 & 653 & 634 & 603 & 626 & 619 \\
\hline $\mathrm{T}_{11}: \mathrm{T}_{3}+$ gypsum @ $5 \mathrm{q}^{-1}$ & 599 & 638 & 619 & 581 & 617 & 594 \\
\hline SEm \pm & 16.39 & 17.58 & 19.55 & 16.49 & 18.87 & 15.38 \\
\hline $\mathrm{CD}(0.05)$ & 48 & 51 & 57 & 48 & 55 & 45 \\
\hline $\mathrm{CV}(\%)$ & 5.0 & 5.6 & 6.5 & 5.2 & 5.9 & 5.2 \\
\hline
\end{tabular}

Table.7 Effect of long-term use of manures and fertilizers on yield of cotton

\begin{tabular}{|c|c|c|c|c|c|c|}
\hline \multirow{2}{*}{ Treatments } & \multirow{2}{*}{$\begin{array}{c}\text { Seed } \\
\text { cotton } \\
\text { yield } \\
\text { kg ha }^{-1}\end{array}$} & \multirow{2}{*}{$\begin{array}{c}\% \\
\text { increase } \\
\text { over } \\
\text { control }\end{array}$} & \multirow{2}{*}{$\begin{array}{c}\text { \% increase } \\
\text { (or) } \\
\text { decrease } \\
\text { over } \mathrm{T}_{3}\end{array}$} & $\begin{array}{l}\text { Stalk } \\
\text { yield }\end{array}$ & $\begin{array}{l}\text { Biological } \\
\text { yield }\end{array}$ & \multirow{2}{*}{$\underset{(\%)}{\text { HI }}$} \\
\hline & & & & \multicolumn{2}{|c|}{$\mathrm{kg} \mathrm{ha}^{-1}$} & \\
\hline $\mathrm{T}_{1}$ : Control & 939 & - & -45.9 & 1836 & 2775 & 33.8 \\
\hline $\mathrm{T}_{2}: 50 \% \mathrm{RD}$ of NPK & 1370 & 46.0 & -21.0 & 2696 & 4066 & 33.7 \\
\hline $\mathrm{T}_{3}: 100 \% \mathrm{RD}$ of $\mathrm{NPK}$ & 1735 & 84.8 & - & 3434 & 5169 & 33.5 \\
\hline $\mathrm{T}_{4}: 150 \% \mathrm{RD}$ of NPK & 2135 & 127.4 & 23.0 & 4228 & 6363 & 33.5 \\
\hline $\mathrm{T}_{5}: 100 \% \mathrm{RD}$ of NP & 1527 & 62.6 & -11.9 & 3026 & 4553 & 33.5 \\
\hline $\mathrm{T}_{6}: 100 \% \mathrm{RD}$ of $\mathrm{N}$ & 1399 & 49.0 & -19.4 & 2765 & 4164 & 33.6 \\
\hline $\mathrm{T}_{7}: \mathrm{T}_{3}+\mathrm{FYM} @ 10 \mathrm{t} \mathrm{ha}^{-1}$ & 2181 & 132.3 & 25.7 & 4330 & 6511 & 33.5 \\
\hline $\mathrm{T}_{8}: \mathrm{T}_{3}+\mathrm{ZnSO}_{4} @ 50 \mathrm{~kg} \mathrm{ha}^{-1}$ & 1806 & 92.3 & 4.0 & 3586 & 5393 & 33.5 \\
\hline $\mathrm{T}_{9}: \mathrm{T}_{3}+\mathrm{MgSO}_{4} @ 50 \mathrm{kgha}^{-1}$ & 1797 & 91.4 & 3.6 & 3560 & 5359 & 33.5 \\
\hline $\mathrm{T}_{10}: 200 \% \mathrm{RD}$ of NPK & 1980 & 110.9 & 14.1 & 3913 & 5893 & 33.6 \\
\hline $\mathrm{T}_{11}: \mathrm{T}_{3}+$ gypsum @ $9 \mathrm{qha}^{-1}$ & 1862 & 98.3 & 7.3 & 3686 & 5548 & 33.5 \\
\hline $\mathrm{SEm} \pm$ & 104.9 & - & - & 173.8 & 260.7 & 0.02 \\
\hline $\mathrm{CD}(0.05)$ & 316 & - & - & 512 & 769 & NS \\
\hline $\mathrm{CV}(\%)$ & 11.2 & - & - & 8.9 & 8.9 & 0.08 \\
\hline
\end{tabular}


Table.8 Effect of long-term use of manures and fertilizers on nutrient content (\%) of cotton

\begin{tabular}{|c|c|c|c|c|c|c|}
\hline \multirow{2}{*}{ Treatments } & \multicolumn{2}{|c|}{ Nitrogen } & \multicolumn{2}{|c|}{ Phosphorus } & \multicolumn{2}{|c|}{ Potassium } \\
\hline & Flowering & Harvest & Flowering & Harvest & Flowering & Harvest \\
\hline $\mathrm{T}_{1}$ : Control & 2.36 & 2.22 & 0.36 & 0.26 & 2.83 & 2.28 \\
\hline $\mathrm{T}_{2}: 50 \% \mathrm{RD}$ of NPK & 2.55 & 2.30 & 0.44 & 0.32 & 2.90 & 2.43 \\
\hline $\mathrm{T}_{3}: 100 \% \mathrm{RD}$ of NPK & 2.66 & 2.45 & 0.51 & 0.40 & 3.09 & 2.50 \\
\hline $\mathrm{T}_{4}: 150 \% \mathrm{RD}$ of NPK & 2.82 & 2.63 & 0.60 & 0.47 & 3.22 & 2.65 \\
\hline $\mathrm{T}_{5}: 100 \% \mathrm{RD}$ of NP & 2.60 & 2.42 & 0.50 & 0.38 & 3.02 & 2.44 \\
\hline $\mathrm{T}_{6}: 100 \% \mathrm{RD}$ of $\mathrm{N}$ & 2.48 & 2.40 & 0.47 & 0.34 & 2.97 & 2.38 \\
\hline $\mathrm{T}_{7}: \mathrm{T}_{3}+\mathrm{FYM} @ 10 \mathrm{th}^{-1}$ & 3.00 & 2.77 & 0.63 & 0.50 & 3.31 & 2.73 \\
\hline $\mathrm{T}_{8}: \mathrm{T}_{3}+\mathrm{ZnSO}_{4} @ 50 \mathrm{kgha}^{-1}$ & 2.68 & 2.48 & 0.53 & 0.42 & 3.14 & 2.53 \\
\hline $\mathrm{T}_{9}: \mathrm{T}_{3}+\mathrm{MgSO}_{4} @ 50 \mathrm{~kg} \mathrm{ha}^{-1}$ & 2.69 & 2.47 & 0.52 & 0.41 & 3.10 & 2.51 \\
\hline $\mathrm{T}_{10}: 200 \% \mathrm{RD}$ of NPK & 2.78 & 2.60 & 0.57 & 0.44 & 3.15 & 2.60 \\
\hline $\mathrm{T}_{11}: \mathrm{T}_{3}+$ gypsum @ $5 \mathrm{q} \mathrm{ha}^{-1}$ & 2.70 & 2.52 & 0.54 & 0.40 & 3.17 & 2.54 \\
\hline SEm \pm & 0.08 & 0.08 & 0.02 & 0.03 & 0.09 & 0.09 \\
\hline $\mathrm{CD}(0.05)$ & 0.25 & 0.23 & 0.06 & 0.09 & 0.26 & NS \\
\hline $\mathrm{CV}(\%)$ & 5.5 & 5.4 & 6.9 & 13.4 & 5.1 & 5.9 \\
\hline
\end{tabular}

Table.9 Effect of long-term use of manures and fertilizers on nutrient uptake

\begin{tabular}{|c|c|c|c|c|c|c|}
\hline \multirow{2}{*}{ Treatments } & \multicolumn{2}{|c|}{ Nitrogen } & \multicolumn{2}{|c|}{ Phosphorus } & \multicolumn{2}{|c|}{ Potassium } \\
\hline & Flowering & Harvest & Flowering & Harvest & Flowering & Harvest \\
\hline $\mathrm{T}_{1}$ : Control & 42.7 & 61.5 & 6.5 & 7.0 & 50.7 & 63.1 \\
\hline $\mathrm{T}_{2}: 50 \% \mathrm{RD}$ of NPK & 67.5 & 93.8 & 11.6 & 13.1 & 76.8 & 99.0 \\
\hline $\mathrm{T}_{3}: 100 \% \mathrm{RD}$ of NPK & 90.3 & 126.9 & 17.3 & 20.7 & 104.7 & 129.4 \\
\hline $\mathrm{T}_{4}: 150 \% \mathrm{RD}$ of NPK & 118.2 & 171.8 & 25.1 & 30.3 & 134.6 & 171.9 \\
\hline $\mathrm{T}_{5}: 100 \% \mathrm{RD}$ of NP & 76.9 & 110.1 & 14.7 & 17.2 & 90.9 & 110.5 \\
\hline $\mathrm{T}_{6}: 100 \% \mathrm{RD}$ of $\mathrm{N}$ & 67.4 & 99.8 & 12.7 & 14.1 & 80.9 & 99.2 \\
\hline $\mathrm{T}_{7}: \mathrm{T}_{3}+\mathrm{FYM} @ 10 \mathrm{tha}^{-1}$ & 128.7 & 176.5 & 26.9 & 31.6 & 141.9 & 173.9 \\
\hline $\mathrm{T}_{8}: \mathrm{T}_{3}+\mathrm{ZnSO}_{4} @ 50 \mathrm{kgha}^{-1}$ & 94.9 & 133.7 & 18.7 & 22.1 & 111.1 & 136.5 \\
\hline $\mathrm{T}_{9}: \mathrm{T}_{3}+\mathrm{MgSO}_{4} @ 50 \mathrm{kgha}^{-1}$ & 94.7 & 132.4 & 18.3 & 21.9 & 109.0 & 134.6 \\
\hline $\mathrm{T}_{10}: 200 \% \mathrm{RD}$ of NPK & 107.5 & 153.5 & 22.1 & 25.7 & 121.8 & 152.9 \\
\hline $\mathrm{T}_{11}: \mathrm{T}_{3}+$ gypsum @ $5 \mathrm{q} \mathrm{ha}^{-1}$ & 98.4 & 140.1 & 19.6 & 22.7 & 115.5 & 141.1 \\
\hline SEm \pm & 5.01 & 8.30 & 1.03 & 1.87 & 6.43 & 6.98 \\
\hline $\mathrm{CD}(0.05)$ & 14.8 & 24.5 & 3.0 & 5.5 & 18.9 & 20.6 \\
\hline CV (\%) & 9.7 & 11.3 & 10.1 & 15.7 & 10.8 & 9.4 \\
\hline
\end{tabular}

Table.10 Data of initial soil samples (1991)

\begin{tabular}{|l|l|c|}
\hline S. No. & \multicolumn{1}{|c|}{ Soil Properties } & Soil values \\
\hline 1 & $\mathrm{pH}$ & 8.4 \\
\hline 2 & $\mathrm{EC} \mathrm{dS} \mathrm{m}$ & -1 \\
\hline 3 & $\mathrm{OC}(\%)$ & 0.60 \\
\hline 4 & Available nitrogen $\left(\mathrm{kg} \mathrm{ha}^{-1}\right)$ & 0.37 \\
\hline 5 & Available phosphorus $\left(\mathrm{kg} \mathrm{P}_{2} \mathrm{O}_{5} \mathrm{ha}^{-1}\right)$ & 196 \\
\hline 6 & Available potassium $\left(\mathrm{kg} \mathrm{K}_{2} \mathrm{O} \mathrm{ha}^{-1}\right)$ & 23 \\
\hline
\end{tabular}




\section{Stalk yield and biological yield ( $\left.\mathrm{kg} \mathrm{ha}^{-1}\right)$}

The data (Table 7 and Fig. 6) indicated that different nutrient treatments significantly influenced the stalk yield of cotton crop. Control plot $\left(\mathrm{T}_{1}\right)$ showed a drastic reduction in the stalk yield due to the removal and depletion of nutrients with continuous cropping without fertilization (Bharadwaj and Omanwar, 1994). The treatments $T_{8}, T_{9}$ and $\mathrm{T}_{11}$ were on par with each other and recorded significantly lower stalk yield than $\mathrm{T}_{7}$. The highest stalk yield recorded in $\mathrm{T}_{7}$ due to better nutrition of crop plants influenced FYM application which might have increased the photosynthesis rate (Rajarajan et al., 2005).

The biological yield (table 7) (kapas yield + stalk yield) was significantly influenced by application of 100\% RD of NPK+FYM @ 10 $\mathrm{t} \mathrm{ha}^{-1}\left(\mathrm{~T}_{7}\right)$ over control. The highest biological yield was observed in $T_{7}$ followed by $\mathrm{T}_{4}$ and $\mathrm{T}_{10}$. Overall, the highest biological yield recorded in combined treatment was attributed to the synergistic interaction primarily effect between FYM and inorganic fertilizers. FYM (farm yard manure) might have acted as a source of additional nutrients and moisture retention.

\section{Nitrogen, phosphorus and potassium content in cotton at harvest stages}

At harvest stage, the highest $\mathrm{N}, \mathrm{P}$ and $\mathrm{K}$ content in plant (Table 8) was noticed in $\mathrm{T}_{7}$ $\left(100 \%\right.$ NPK +10 t FYM ha $\left.^{-1}\right)$ but N and P content were found at par with $150 \% \mathrm{RD}$ of NPK and 200\% RD of NPK treatments. Increasing dose of inorganic fertilizers showed enhanced primary nutrient content in cotton upto $150 \%$ RD of NPK. The treatments $100 \% \mathrm{NPK}+\mathrm{ZnSO}_{4} @ 50 \mathrm{~kg} \mathrm{ha}^{-1}\left(\mathrm{~T}_{8}\right), 100 \%$ $\mathrm{NPK}+\mathrm{MgSO}_{4} @ 50 \mathrm{~kg} \mathrm{ha}^{-1}\left(\mathrm{~T}_{9}\right)$ and 100\% $\mathrm{RD}$ of NPK + gypsum $5 \mathrm{q} \mathrm{ha}^{-1}\left(\mathrm{~T}_{11}\right)$ recorded more nitrogen content in cotton plant over $100 \%$ RD of NPK treatment $\left(\mathrm{T}_{3}\right)$. The higher nutrient content resulted on integrated management of organic and inorganic sources $\left(\mathrm{T}_{7}\right)$ might be the cause for increased concentration of nutrients mainly nitrogen in cotton (Nawlakhe and Mankar, 2011).

Phosphorus content in cotton was highest $(0.50 \%)$ in treatment $\mathrm{T}_{7}$ that received $(100 \%$ RD of NPK + FYM @ $\left.10 \mathrm{t} \mathrm{ha}^{-1}\right)$. The increased content of phosphorus with conjunctive use of FYM and inorganics might be due to the formation of phosphohumic complexes, which were more easily assimilated by the plants.

The highest $\mathrm{K}$ content was recorded in the treatment $\mathrm{T}_{7}$ (100 per cent recommended dose of NPK+FYM @ $10 \mathrm{t} \mathrm{ha}^{-1}$ ) with 2.73 per cent followed by $\mathrm{T}_{4}$ (150 per cent recommended dose of NPK) with 2.65 per cent.

Continuous manuring and fertilization at the same site for long period affected the soil fertility and there by uptake of nutrient by crop (Table 9). In this present study increasing trend in uptake of NPK by cotton was observed with increasing fertilizer levels from 50 to 150 per cent of RDF and slightly decreased at $200 \%$ RDF. The highest uptake of $\mathrm{N}, \mathrm{P}$ and $\mathrm{K}\left(176.5,31.6\right.$ and $173.9 \mathrm{~kg} \mathrm{ha}^{-1}$ respectively) were recorded with the application of $100 \%$ RD of NPK + FYM $10 \mathrm{t}$ $\mathrm{ha}^{-1}$ followed by $150 \%$ RD of NPK $\left(\mathrm{T}_{4}\right)$.

The lowest total uptake of $\mathrm{N}, \mathrm{P}$ and $\mathrm{K}$ were observed i.e. 61.5, 7.0 and $63.1 \mathrm{~kg} \mathrm{ha}^{-1}$ respectively by cotton in control treatment. Application of super optimal dose $\left(\mathrm{T}_{4}, 150 \%\right.$ RD of NPK) of treatment showed significant increase in $\mathrm{N}, \mathrm{P}$ and $\mathrm{K}$ uptake by cotton over $50 \%, 100 \%$ RDF and control.

Finally, it can be concluded that combined use of organics and inorganics can help not only in increasing the yield and uptake of nutrients but also in improving soil organic 
carbon, available nutrient status and thereby increasing the nutrient supplying capacity of soil, which in turn help in sustainable crop production.

\section{References}

Ahmed, A. U. H., R. Ali, S. I. Zamir and Mehmood, N. (2009). Growth, yield and quality performance of cotton cultivar BH-160 (Gossypium hirsutum L.). The Journal of Animal and Plant Sciences. 19(4):189-192.

Bandyopadhyay, K.K., Ghosh, P.K., Chaudhary, R.S., Mati, K., Mandal, K.G and Mishra, A.K. 2004. Integrated nutrient management practices in soybean and sorghum in sole and intercropping system in a Vertisol. Indian Journal of Agricultural Science. 74: 55-63.

Bharadwaj, V and Omanwar, P.K. 1994. Long-term effect of continuous rotational cropping and fertilisation on crop yields and soil properties-II. Effects on EC, $\mathrm{pH}$, organic matter and available nutrients of soil. Journal of the Indian Society of Soil Science. 42: 387-392.

Biswas, T. D and Khosla, B. K. 1971. Building up of organic matter status of the soil and its relation to the soil physical properties. Proceedings of International Symposium on Soil Fertility Evaluation. New Delhi, pp. 831-842.

Chaudhary, D.R., Arupghosh, Sharma, M.K and Chikara, J. 2006. Characterization of some salt affected soils of Amethi, Uttar Pradesh. Agropedology.16: 126129.

Giora J. K., Arnon, K and Itzhak, B. 2010. Degradation of soil fertility following cycles of cotton-cereal cultivation in Mali, West Africa: A first approximation to the problem. Soil and
Tillage Research. 106: 254-262.

Hirekurabar, P.M., Satyanarayana, T., Sarangnati, P.A and Manjunatham, H.M. 2000. Forms of potassium and their distribution in soils under cottonbased cropping system in Karnataka. Journal of the Indian Society of Soil Science. 48: 604-608.

Jackson, M. L. 1973. Soil Chemical Analysis. Prentice Hall of India Private Ltd., New Delhi: 134-182.

Lalithakumari, A., Veeraiah, $\mathrm{K}$ and Ratnakumari, S. 2010. Long-term effects of manures and fertilizers on productivity of rainfed cotton and soil fertility in Vertisols. Journal of Cotton Research and Development. 24: 200204.

Muhr, G R., Datta, N.P., Sankarasubramoney, H., Leley, V.K and Dunabha, R.L. 1965. Soil testing in India. $2^{\text {nd }}$, USAID - Mission to India, New Delhi.

Nambiar, K.K. Mandal and Abrol, I.P.1989. Long-term fertilizer experiments in India -an over view. Fertilizer News. 34: 11-20.

Nawlakhe, S.M and Mankar, D.D. 2011. Nitrogen uptake in cotton + greengram intercropping system influenced by integrated nutrient management. Crop Research. 41: 59-63.

Olsen, S.R., Cole, C.V., Watanabe, F.S and Dean, L.A. 1954. Estimation of available phosphorus in soils by extraction with sodium bicarbonate. Circular of United States Development of Agriculture. pp.939.

Piper, C.S. 1966. Soil and Plant Analysis. Hans Publishers, Bombay. pp: 368.

Prasad, J., Karmakar, S., Kumar, R and Mishra, B. 2010a. Influence of integrated nutrient management on yield and soil properties in maize-wheat cropping system in an Alfisol of Jharkhand. Journal of the Indian Society of Soil Science, 58: 200-204. 
Prasad, R.K., Vipinkumar, Prasad, B and Singh, A.P. 2010b. Long-term effect of crop residues and zinc fertilizer on crop yield, nutrient uptake and fertility build up under rice wheat cropping system in Calciorthents. Journal of the Indian Society of Soil Science. 58 (2): 205-211.

Rajarajan, A., Janaki. P., Appavu, A and Vadivel, A. 2005. Effect of fertilizer NPK and FYM on yields of cotton and nutrient status in black soil. Madras Agricultural Journal. 92: 266-270.

Sahadevareddy, B and Aruna, E. 2008. Integrated nutrient management in hybrid cotton. Journal of Cotton Research and Development. 22: 153156.
Sawan, Z. M., Mahmoud M. H and ElGuibali, A. H. (2006). Response of yield, yield components, and fiber properties of Egyptian cotton (Gossypium barbadense L.) to nitrogen fertilization and foliar-applied potassium and mepiquat chloride. The Journal of Cotton Science. 10:224-234.

Sinha, S.K., Singh, V.N and Singh, P. 1997. Effect of continuous use of fertilisers on physical and physico-chemical properties of an alluvial soil. Journal of Research (BAU), 9: 31-34.

Subbiah, B.V and Asija, C.L. 1956. A rapid procedure for the estimation of available nitrogen in soils. Current Science. 25: 259-260.

\section{How to cite this article:}

Joga Rao, P., P.R.K. Prasad, A. Lalitha Kumari, P. Prasuna Rani and Pulla Rao, C.H. 2017. Long-Term Effect of Manures and Fertilizers on Nutrient Status under Cotton Mono-Cropping in Vertisol. Int.J.Curr.Microbiol.App.Sci. 6(7): 2084-2094.

doi: https://doi.org/10.20546/ijcmas.2017.607.246 